\title{
ON CERTAIN OPTIMAL DIFFEOMORPHISMS BETWEEN CLOSED CURVES
}

\author{
ANDREA CERRI AND BARBARA DI FABIO
}

\begin{abstract}
The concept of natural pseudo-distance has proven to be a powerful tool for measuring the dissimilarity between shape properties of topological spaces, modeled as continuous real-valued functions defined on the spaces themselves. Roughly speaking, the natural pseudo-distance is defined as the infimum of the change of the functions' values, when moving from one space to the other through homeomorphisms, if possible. In this paper, we prove the first available result about the existence of optimal homeomorphisms between closed curves, i.e. inducing a change of the function that equals the natural pseudo-distance. Moreover, we show that, under our assumptions, this optimal homeomorphism is actually a diffeomorphism.
\end{abstract}

\section{INTRODUCTION}

Formalizing the concept of shape for topological spaces and manifolds, as well as providing an efficient comparison of shapes, has been a widely researched topic in the last decade. As such, a class of methods has been developed with the purpose of performing a topological exploration of shapes, according to some quantitative geometric properties provided by a real function chosen to extract shape features $[1,3,19,22,29]$.

In this context, Size Theory was introduced at the beginning of the 1990s [14, $15,16]$, supported by the adoption of a suitable mathematical tool: the natural pseudo-distance $[10,11,12]$.

In the formalism of Size Theory, a shape is modeled as a pair $(X, \varphi)$, where $X$ is a topological space and $\varphi: X \rightarrow \mathbb{R}$ is a continuous function $[1,16]$. Such a pair is called a size pair and $\varphi$ is called a measuring function. The role of $\varphi$ is to take into account only the properties considered relevant for the shape comparison problem at hand, while disregarding the irrelevant ones, as well as to impose the desired invariance properties (e.g., invariance with respect to isometries, or affine, or projective transformations).

The natural pseudo-distance $\delta$ measures the dissimilarity between two size pairs $(X, \varphi),(Y, \psi)$. Roughly speaking, it is defined as the infimum of the variation of the values of $\varphi$ and $\psi$, when we move from $X$ to $Y$ through homeomorphisms, if possible (see Definition 1.2). Therefore, two size pairs "have the same shape" if they share the same shape properties, expressed by the measuring functions' values, that is, their natural pseudo-distance vanishes.

Date: April 28, 2011.

2010 Mathematics Subject Classification. Primary 57S05, 57S10; Secondary 54C30, 68U05.

Key words and phrases. Natural pseudo-distance, measuring function, Morse function, Size Theory.

A.C. was supported in part by the Austrian Science Fund (FWF) grant no. P20134-N13. 
Earlier results about the natural pseudo-distance can be divided into two classes. One class gives information on the possible values assumed by the natural pseudodistance $\delta$ between two size pairs $(X, \varphi),(Y, \psi)$. For example, if the considered topological spaces $X$ and $Y$ are smooth closed manifolds and the measuring functions are also smooth, then it holds that $\delta((X, \varphi),(Y, \psi))=\frac{C}{k}$, with $C$ the distance between two critical values of the functions $\varphi$ and $\psi$, and $k$ a suitable positive integer number [10]. In particular, the value of $k$ can only be either 1 or 2 in the case of curves [12], while it cannot be greater than 3 in the case of surfaces [11].

The other class of results provides estimations of the natural pseudo-distance $[4,9,18]$, with particular reference to the use of the so-called size functions $[6,9]$. Size functions are shape descriptors containing information about the considered size pairs, and admitting a discrete and complete representation by means of certain countable sets of points in the real plane $[13,17,23]$. Comparing these sets of points induces a distance between the associated size functions, which has proven to be a lower bound for the natural pseudo-distance between the considered size pairs [6]. In this way, it is possible to obtain information about the natural pseudo-distance without actually computing it. The research on size functions has led to a formal setting, which has turned out to be useful, not only from a theoretical point of view, but also on the application side (see, e.g., [2, 5, 8, 27, 28]).

The contribution of this paper. Besides being a useful theoretical tool for applications in shape comparison, the natural pseudo-distance is challenging from the mathematical point of view, and several questions about its properties need further investigation. One among them consists in establishing the conditions ensuring the existence of optimal homeomorphisms between size pairs, i.e. homeomorphisms realizing the natural pseudo-distance (cf. Definition 1.3). It is possible to show that, in general, such homeomorphisms do not exist (see, e.g., Section 2).

In this paper, we provide the first available result about the existence of optimal homeomorphisms (Theorem 3.4). To be more precise, our result shows that, if the considered spaces are closed curves (i.e. compact and without boundary 1manifolds) endowed with Morse measuring functions such that the natural pseudodistance is zero, it is possible to construct a point-to-point match between such curves, that is optimal in the sense that it does not change the measuring functions' values. In addiction, we show that, under our assumptions, this point-to-point match is actually a diffeomorphism. In the language of Size Theory, our result means that the considered curves share the same shape properties with respect to the chosen measuring functions. Moreover, from a topological point of view, our theorem is equivalent to claim that it is possible to quotient the space of Morse functions on the circle $S^{1}$ in such a way that the equivalence classes are closed (see Section 3.1).

In the initial contribution to establishing stability properties of Reeb graphs proposed in [7], our result has been used to prove the positive definiteness of an editing distance between Reeb graphs of closed curves. This editing distance results to be suitable to compare Reeb graphs shape descriptors for curves. Indeed, it has been shown that changes in functions imply smaller changes in the editing distance. This proves stability of Reeb graphs of curves under function perturbations.

In general, the subject of our work fits in the current mathematical research and interest in simple closed curves, motivated by problems concerning shape comparison and matching in Computer Vision. For example, in [24] and [25] the authors 
analyse (from a mathematical point of view) the (dis)similarity judgement induced by human perception in comparing 2-dimensional shapes. Modeling these shapes as compact, simply connected planar regions bounded by simple closed curves, they are led to study Riemannian metrics on the space of closed curves. In [20] and [21] the author, inspired by problems coming from applied sciences, studies certain correspondences (the so-called bimorphisms) between simple closed planar curves, in order to model in a rigorous way the concept of "optimal match" between these objects. Indeed, the problem of curve matching is a lively research topic in disciplines such as Image Analysis, Image Comparison and Pattern Recognition. In these contexts, matching two closed curves allows, for example, the comparison of two planar images by taking into account their silhouettes or contour curves, with applications, e.g., in medicine, cognitive science, and information technology.

Outline. The paper is divided into three sections. Section 1 deals with some of the standard facts on the comparison of size pairs via the natural pseudo-distance. In particular, the definition of the natural pseudo-distance $\delta$ and its main properties are recalled, focusing on the concepts of optimal homeomorphism and $d$ approximating sequence. Section 2 is devoted to the description of some simple and clarifying examples showing that all the conditions we require in stating our main result are necessary. In Section 3 we prove our main result concerning the existence and the construction of an optimal $C^{2}$-diffeomorphism between two smooth closed curves endowed with Morse measuring functions (Theorem 3.4).

\section{Preliminaries}

In Size Theory, a size pair is a pair $(X, \varphi)$, where $X$ is a non-empty, compact, locally connected Hausdorff space and $\varphi: X \rightarrow \mathbb{R}$ is a continuous function called a measuring function. Let Size be the collection of all the size pairs, and let $(X, \varphi),(Y, \psi)$ be two size pairs. We denote by $H(X, Y)$ the set of all homeomorphisms from $X$ to $Y$.

Definition 1.1. If $H(X, Y) \neq \emptyset$, the function $\Theta: H(X, Y) \rightarrow \mathbb{R}$ given by

$$
\Theta(f)=\max _{x \in X}|\varphi(x)-\psi(f(x))|
$$

is called the natural size measure with respect to the measuring functions $\varphi$ and $\psi$.

Roughly speaking, $\Theta(f)$ measures how much $f$ changes the values taken by the measuring functions, at corresponding points.

Definition 1.2. We shall call natural pseudo-distance the (extended) pseudodistance $\delta:$ Size $\times$ Size $\rightarrow \mathbb{R} \cup\{+\infty\}$ defined as

$$
\delta((X, \varphi),(Y, \psi))= \begin{cases}\inf _{f \in H(X, Y)} \Theta(f), & \text { if } H(X, Y) \neq \emptyset \\ +\infty, & \text { otherwise. }\end{cases}
$$

Note that $\delta$ is not a distance, since two different size pairs $(X, \varphi),(Y, \psi)$ can have a vanishing pseudo-distance. In that case, $X$ and $Y$ are only sharing the same shape properties with respect to the chosen functions $\varphi$ and $\psi$, respectively.

A simple example shows this fact. Let us consider the circle $X=\{(u, v) \in$ $\left.\mathbb{R}^{2}: u^{2}+v^{2}=1\right\}$ and the ellipse $Y=\left\{(u, v) \in \mathbb{R}^{2}: a u^{2}+v^{2}=1\right\}$ for a fixed real number $a>0, a \neq 1$. Moreover, let us endow $X$ and $Y$ with the measuring functions $\varphi=\xi_{\left.\right|_{X}}$ and $\psi=\xi_{\left.\right|_{Y}}$, respectively, where $\xi: \mathbb{R}^{2} \rightarrow \mathbb{R}$ is the continuous 
function taking each $(u, v) \in \mathbb{R}^{2}$ to its ordinate $v$. In this case, it can be proved that $\delta((X, \varphi),(Y, \psi))=0$. Indeed, it is easy to check that a homeomorphism exists deforming $X$ into $Y$ without changing the points' ordinates.

On the other hand, if we endow $X$ and $Y$ with the functions $\hat{\varphi}=\hat{\xi}_{\left.\right|_{X}}$ and $\hat{\psi}=\hat{\xi}_{\left.\right|_{Y}}$, respectively, where $\hat{\xi}: \mathbb{R}^{2} \rightarrow \mathbb{R}$ is the continuous function taking each $(u, v) \in \mathbb{R}^{2}$ to its Euclidean distance from the axis origin, it follows that $\delta((X, \hat{\varphi}),(Y, \hat{\psi}))=$ $\left|\frac{1}{\sqrt{a}}-1\right|>0$.

Therefore, in our framework, we can say that $X$ and $Y$ have the same "shape" if compared with respect to their height, while they have a different "shape" when considered in terms of the distance from their center of mass.

Definition 1.3. Let $(X, \varphi),(Y, \psi)$ be two size pairs with $H(X, Y) \neq \emptyset$. We shall say that $f \in H(X, Y)$ is an optimal homeomorphism if $\Theta(f)=\delta((X, \varphi),(Y, \psi))$.

Let us observe that such a homeomorphism may not exist, and even when it exists, it is not straightforward to define it. On the other hand, Definition 1.2 implies that, if $H(X, Y) \neq \emptyset$, we can always find a sequence $\left(f_{k}\right)$ of homeomorphisms from $X$ to $Y$ such that $\lim _{k \rightarrow \infty} \Theta\left(f_{k}\right)=\delta((X, \varphi),(Y, \psi))$.

Definition 1.4. Let $(X, \varphi),(Y, \psi)$ be two size pairs with $\delta((X, \varphi),(Y, \psi))=d<\infty$. Every sequence $\left(f_{k}\right)$ of homeomorphisms $f_{k}: X \rightarrow Y$ such that $\lim _{k \rightarrow \infty} \Theta\left(f_{k}\right)=d$ is said to be a $d$-approximating sequence from $(X, \varphi)$ to $(Y, \psi)$.

Remark 1.5. We observe that $\left(f_{k}\right)$ is a d-approximating sequence from $(X, \varphi)$ to $(Y, \psi)$ if and only if $\left(f_{k}^{-1}\right)$ is a d-approximating sequence from $(Y, \psi)$ to $(X, \varphi)$.

The main goal of this paper is to show that an optimal differentiable of class $C^{2}$ homeomorphism (from now on an optimal $C^{2}$-diffeomorphism, or simply an optimal diffeomorphism) exists between two size pairs $(X, \varphi)$ and $(Y, \psi)$, under the following conditions:

(a) $\delta((X, \varphi),(Y, \psi))=0$;

(b) $X$ and $Y$ are two curves;

(c) $\varphi$ and $\psi$ are Morse (i.e., of class $C^{2}$ with invertible Hessian at each critical point [26]) .

This result will be formally given and proved later in the case that the curves are closed (Theorem 3.4). However, we remark that the closedness requirement will be made only for the sake of simplicity. Indeed, it can be weakened to compact 1-manifolds having non-empty boundary, without much affecting the following reasonings.

\section{Some explanatory eXamples}

We provide here three meaningful examples showing that assumptions $(a),(b)$, (c) introduced in Section 1 are necessary to ensure the existence of an optimal diffeomorphism between two size pairs $(X, \varphi)$ and $(Y, \psi)$. Indeed, if one among them is dropped, then an homeomorphism attaining the natural pseudo-distance might not exist, implying that Theorem 3.4 is false.

Without loss of generality, in the examples and figures we describe here, we shall always assume that the spaces $X$ and $Y$ are embedded in $\mathbb{R}^{3}$ and both $\varphi$ and $\psi$ are 
the $z$-coordinate functions, i.e. $\varphi=z_{\left.\right|_{X}}, \psi=z_{\left.\right|_{Y}}$. Moreover, by abuse of notation, we will simply write $\varphi=\psi=z$.

The first two examples we make ready to describe are taken from [10].

Example 1 (Hypothesis (a) fails). This example shows that, if two size pairs satisfy hypotheses $(b)$ and $(c)$, but have a non-vanishing natural pseudo-distance, then an optimal homeomorphism may not exist.

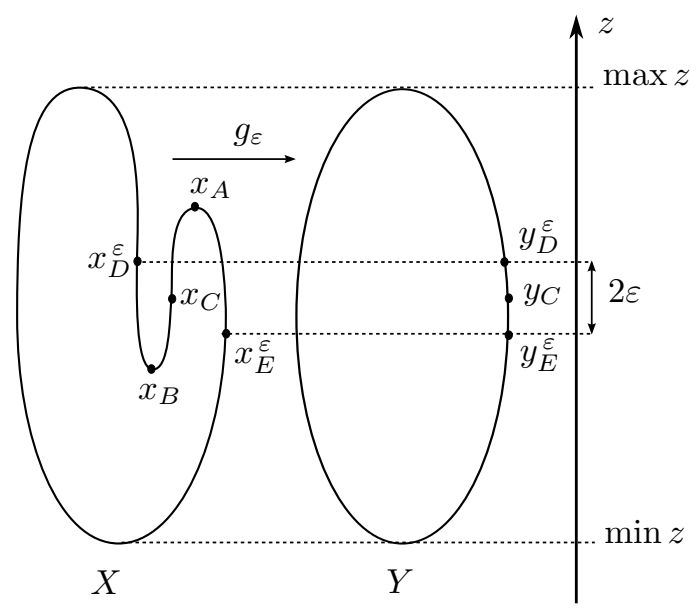

Figure 1. An example of two closed curves $X, Y$ endowed with the Morse function $z$. No optimal homeomorphism exists between $(X, z),(Y, z)$ because their natural pseudo-distance is non-zero.

Let us consider the two size pairs $(X, z),(Y, z)$ depicted in Figure 1, where $X$ and $Y$ are smooth closed curves in $\mathbb{R}^{3}$, embedded in the real plane, endowed with the Morse function $z$.

As can be seen in Figure 1, the points $x_{A}, x_{B} \in X$ are critical points of the function $z$ and $z\left(x_{C}\right)=\frac{1}{2}\left(z\left(x_{A}\right)+z\left(x_{B}\right)\right)=z\left(y_{C}\right)$. In [12] it has been proved that the natural pseudo-distance between homeomorphic smooth closed curves, endowed with Morse measuring functions, is always obtainable in terms of some critical values of the measuring functions. Actually, in this example it is possible to show that the natural pseudo-distance between $(X, z)$ and $(Y, z)$ takes the value $d=\frac{1}{2}\left(z\left(x_{A}\right)-z\left(x_{B}\right)\right)$. On the other hand, it will also be proved that no optimal homeomorphism exists. Indeed, we can construct a sequence of homeomorphisms $\left(f_{k}\right)$, such that $\lim _{k \rightarrow \infty} \Theta\left(f_{k}\right)=\frac{1}{2}\left(z\left(x_{A}\right)-z\left(x_{B}\right)\right)$, and show that $\Theta(f)>\frac{1}{2}\left(z\left(x_{A}\right)-\right.$ $\left.z\left(x_{B}\right)\right)$ for every homeomorphism $f \in H(X, Y)$. The first step consists in proving that, for every $\varepsilon>0$, a homeomorphism $g_{\varepsilon}: X \rightarrow Y$ exists, such that $\Theta\left(g_{\varepsilon}\right) \leq$ $\frac{1}{2}\left(z\left(x_{A}\right)-z\left(x_{B}\right)\right)+2 \varepsilon$. Accordingly, consider the points $x_{D}^{\varepsilon}, x_{E}^{\varepsilon}, y_{D}^{\varepsilon}$ and $y_{E}^{\varepsilon}$ in Figure 1, verifying $z\left(x_{D}^{\varepsilon}\right)=z\left(y_{D}^{\varepsilon}\right)=z\left(x_{C}\right)+\varepsilon$ and $z\left(x_{E}^{\varepsilon}\right)=z\left(y_{E}^{\varepsilon}\right)=z\left(x_{C}\right)-\varepsilon$. We can choose a homeomorphism $g_{\varepsilon}$, taking the arc $x_{D}^{\varepsilon} x_{C} x_{E}^{\varepsilon}$ to the arc $y_{D}^{\varepsilon} y_{C} y_{E}^{\varepsilon}$ in such a way that $g_{\varepsilon}\left(x_{D}^{\varepsilon}\right)=y_{D}^{\varepsilon}$ and $g_{\varepsilon}\left(x_{E}^{\varepsilon}\right)=y_{E}^{\varepsilon}$. Outside the $\operatorname{arc} x_{D}^{\varepsilon} x_{C} x_{E}^{\varepsilon}$ in $X$ define $g_{\varepsilon}$ by mapping, in the unique possible way, every point $x$ to a point $g_{\varepsilon}(x)$ satisfying $z(x)=z\left(g_{\varepsilon}(x)\right)$. For every $k \in \mathbb{N} \backslash\{0\}$ set $f_{k}=g_{\frac{1}{k}}$. It can be easily verified that $\lim _{k \rightarrow \infty} \Theta\left(f_{k}\right)=\frac{1}{2}\left(z\left(x_{A}\right)-z\left(x_{B}\right)\right)$. 
It only remains to prove that $\Theta(f) \leq \frac{1}{2}\left(z\left(x_{A}\right)-z\left(x_{B}\right)\right)$ for no homeomorphism $f \in H(X, Y)$. Indeed, if such a homeomorphism existed, then, for every $x \in X$, we would have $|z(x)-z(f(x))| \leq \frac{1}{2}\left(z\left(x_{A}\right)-z\left(x_{B}\right)\right)$. By replacing $x$ with $x_{A}$ and $x_{B}$, respectively, we obtain that $\frac{1}{2}\left(3 z\left(x_{A}\right)-z\left(x_{B}\right)\right) \geq z\left(f\left(x_{A}\right)\right) \geq \frac{1}{2}\left(z\left(x_{A}\right)+z\left(x_{B}\right)\right)$ and $\frac{1}{2}\left(z\left(x_{A}\right)+z\left(x_{B}\right)\right) \geq z\left(f\left(x_{B}\right)\right) \geq \frac{1}{2}\left(3 z\left(x_{B}\right)-z\left(x_{A}\right)\right)$, respectively. Hence, $z\left(f\left(x_{A}\right)\right) \geq$ $z\left(y_{C}\right) \geq z\left(f\left(x_{B}\right)\right)$. As a consequence, by extending this $f$ to the whole curve, at least one point $x \in X$ could be found such that $|z(x)-z(f(x))|>\frac{1}{2}\left(z\left(x_{A}\right)-z\left(x_{B}\right)\right)$, contradicting our assumption.

Example 2 (Hypothesis (b) fails). This example shows that there may not exist an optimal homeomorphism between two size pairs satisfying hypotheses $(a)$ and (c), but missing hypothesis $(b)$.

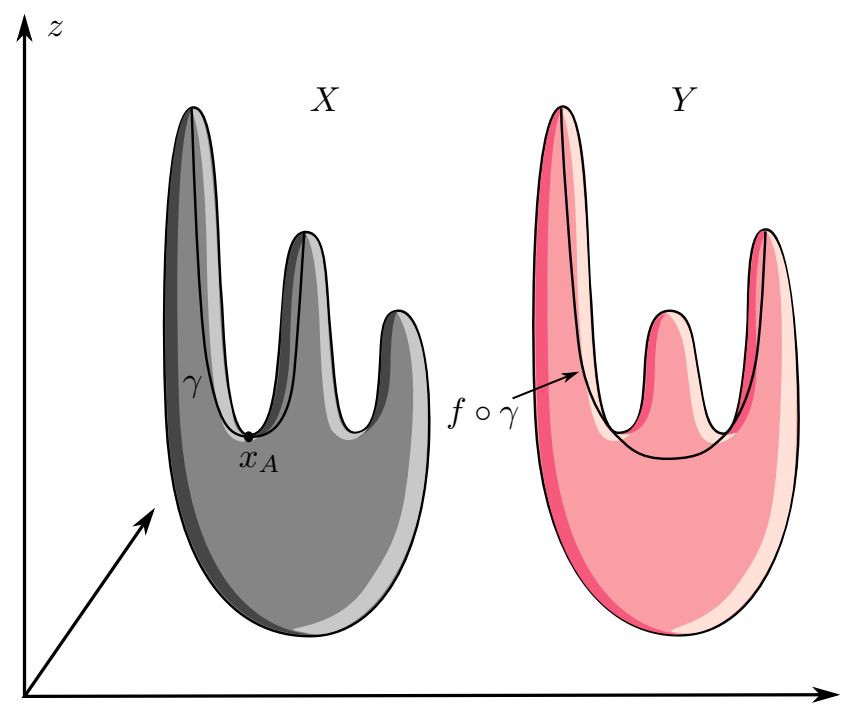

Figure 2. An example of two size pairs $(X, z)$ and $(Y, z)$, whose natural pseudo-distance is zero. No optimal homeomorphism exists between $(X, z)$, $(Y, z)$ because $X$ and $Y$ are not curves.

Consider the smooth surfaces $X$ and $Y$ displayed in Figure 2 and the corresponding measuring function $z$. It is easy to show that the natural pseudo-distance between the two size pairs is zero. Indeed, it is possible to isotopically deform the left surface to the right one by "torsion", exchanging the positions of the two smallest humps. This deformation can be performed by an arbitrarily small change in the values of the $z$-coordinate. Therefore, a sequence of homeomorphisms $\left(f_{k}\right)$ from $X$ to $Y$ can be constructed, such that $\lim _{k \rightarrow \infty} \Theta\left(f_{k}\right)=0$. However, no optimal homeomorphism exists between the two size pairs. Suppose indeed there exists a homeomorphism $f$ such that $\Theta(f)=0$. Consider a path $\gamma$ as in Figure 2, chosen in such a way that, in the image of the path, $z(x)=z\left(x_{A}\right)$ for no point $x \in X$ different from $x_{A}$. It can be easily verified that the image of the path $f \circ \gamma$ has to contain more than one point at which $z$ takes the value $z\left(x_{A}\right)$. This contradicts our assumptions, since $\Theta(f)=0$ implies $z(f(x))=z(x)$ for every $x$ in the image of $\gamma$. 
Example 3 (Hypothesis $(c)$ fails). This last example shows that there may not exist an optimal homeomorphism between two closed curves having vanishing natural pseudo-distance if such curves are endowed with measuring functions missing hypothesis $(c)$.

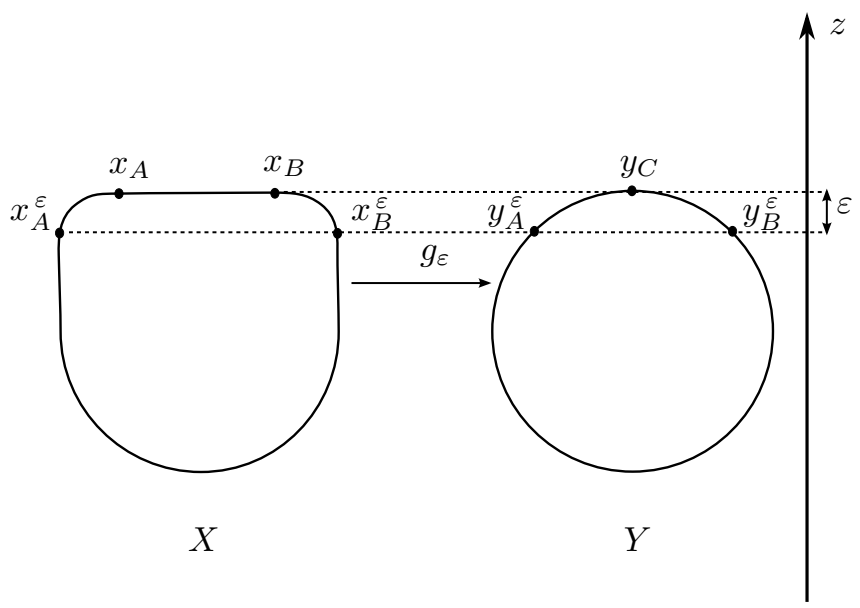

FigURE 3. An example of two size pairs $(X, z)$ and $(Y, z)$, whose natural pseudo-distance is zero. No optimal homeomorphism exists between $(X, z)$, $(Y, z)$ because $z_{\left.\right|_{X}}$ is not Morse.

Let us consider the two size pairs $(X, z)$ and $(Y, z)$ in Figure 3, where $X$ and $Y$ are smooth closed curves. As can be seen, the measuring function $z$ is not Morse on $X$.

We see that the natural pseudo-distance between $(X, z)$ and $(Y, z)$ is vanishing, but an optimal homeomorphism does not exist. Indeed, such a map should verify $\max _{x \in X}|z(x)-z(f(x))|=0$, and therefore it should take each point of the segment $\frac{x \in X}{x_{A} x_{B}}$ to the point $y_{C}$, against the injectivity.

Remark 2.1. The above Example 3 can be generalized to manifolds of any dimension. Indeed, it can be shown that taking a Morse function $\varphi$ on an n-dimensional closed manifold $X, n \geq 1$, it is always possible to find a continuous real-valued function $\psi$ on the same manifold such that the natural pseudo-distance between $(X, \varphi)$ and $(X, \psi)$ is zero, but no optimal homeomorphism of $X$ exists.

\section{MAIN THEOREM}

In this section we prove the main result of this paper (Theorem 3.4). It states that an optimal $C^{2}$-diffeomorphism exists between two closed curves, endowed with Morse measuring functions, and whose natural pseudo-distance is zero. Roughly speaking, the proof involves the idea to construct an optimal homeomorphism $f$ between the two curves as a continuous extension of a bijective map between measuring functions' critical points. Then we show that $f$ is locally a $C^{2}$-diffeomorphism.

Let us now introduce some notations and assumptions we shall adopt in the rest of this section.

Let $(X, \varphi),(Y, \psi)$ be two size pairs, with $X, Y$ two closed curves, and $\varphi, \psi$ Morse measuring functions, and suppose that $\delta((X, \varphi),(Y, \psi))=0$. 
For the sake of simplicity, from now on we shall assume that the considered curves are connected. However, note that this last hypothesis can be weakened to any finite number of connected components, without much affecting the following reasonings.

Let us now consider two parameterizations $h_{X}: S^{1} \rightarrow X, h_{Y}: S^{1} \rightarrow Y$. Considering the clockwise orientation on $S^{1} \subset \mathbb{R}^{2}$, the homeomorphisms $h_{X}, h_{Y}$ allow us to induce an orientation on $X$ and $Y$, respectively. Accordingly, for every two distinct points $x, x^{\prime} \in X$ (respectively $y, y^{\prime} \in Y$ ), we shall denote by $x \curvearrowright x^{\prime}$ (resp. $y \curvearrowright y^{\prime}$ ) the oriented path on $X$ (resp. $Y$ ), induced by $h_{X}$ (resp. $h_{Y}$ ), from the point $x$ (resp. $y$ ) to the point $x^{\prime}$ (resp. $y^{\prime}$ ), going clockwise along $S^{1}$, and including both $x$ and $x^{\prime}$ (resp. $y$ and $y^{\prime}$ ).

Let us consider a 0 -approximating sequence $\left(f_{k}\right)_{k \in \mathbb{N}}$ of homeomorphisms from $(X, \varphi)$ to $(Y, \psi)$, i.e. such that $\lim _{k \rightarrow \infty} \Theta\left(f_{k}\right)=0$. Since homeomorphisms between closed curves can be orientation-preserving or not, for the sake of simplicity we shall assume (possibly by considering a subsequence of $\left(f_{k}\right)$ ) that the orientation is maintained by each $f_{k}$. Indeed, if this is not the case, we can consider a new parametrization $\hat{h}_{Y}$ inducing an orientation that is opposite to the one induced by $h_{Y}$.

Let $\left(x_{1}, \ldots, x_{n}\right), n \geq 2$, be the ordered set of critical points of $\varphi$, taken in $X$ following the orientation induced by $h_{X}$, and starting from $x_{1}$ arbitrarily chosen. Obviously, they are even in number, and correspond, alternatively, to the minima and maxima of $\varphi$ on $X$. From the compactness of $X$ and $Y$, we can assume (possibly by considering a subsequence of $\left(f_{k}\right)$ ) that there exists $\lim _{k \rightarrow \infty} f_{k}\left(x_{i}\right)$ for every $i=1, \ldots, n$. Indeed, since $\left(f_{k}\left(x_{1}\right)\right)$ is a bounded sequence, we can assume (possibly by considering a subsequence) that it converges on $Y$. But $\left(f_{k}\left(x_{2}\right)\right.$ ) is also a bounded sequence, therefore we can also assume (possibly by considering a subsequence) that it converges on $Y$. By applying recursively the same reasoning, we then get that there exists $\lim _{k \rightarrow \infty} f_{k}\left(x_{i}\right)$ for every $i=1, \ldots, n$.

Let us denote $\lim _{k \rightarrow \infty} f_{k}\left(x_{i}\right)$ by $y_{i}$ for every $i=1, \ldots, n$.

Remark 3.1. For every $x \in X$ such that there exists $\lim _{k \rightarrow \infty} f_{k}(x)$, the equality $\varphi(x)=\psi\left(\lim _{k \rightarrow \infty} f_{k}(x)\right)$ holds. Indeed, since $\left(f_{k}\right)$ is a 0-approximating sequence, $\left|\varphi(x)-\psi\left(\lim _{k \rightarrow \infty} f_{k}(x)\right)\right|=\lim _{k \rightarrow \infty}\left|\varphi(x)-\psi\left(f_{k}(x)\right)\right|=0$.

Let us now prove that $y_{1}, \ldots, y_{n}$ are $n$ distinct points of $Y$.

Lemma 3.2. For every $i, j \in\{1, \ldots, n\}$, if $y_{i}=y_{j}$, then $x_{i}=x_{j}$.

Proof. Let $y_{i}=y_{j}$. By contradiction, let us assume that $x_{i} \neq x_{j}$. Then, for every $k \in \mathbb{N}, f_{k}\left(x_{i}\right) \neq f_{k}\left(x_{j}\right)$, and $\lim _{k \rightarrow \infty} f_{k}\left(x_{i}\right)=\lim _{k \rightarrow \infty} f_{k}\left(x_{j}\right)$. Moreover, as a consequence of Remark 3.1, $\varphi\left(x_{i}\right)=\psi\left(y_{i}\right)=\psi\left(y_{j}\right)=\varphi\left(x_{j}\right)$. Since $\varphi$ is Morse, it is necessarily non-constant on the path $x_{i} \curvearrowright x_{j}$. Therefore there exists at least one point $\bar{x} \in x_{i} \curvearrowright x_{j}$, such that $\left|\varphi(\bar{x})-\varphi\left(x_{i}\right)\right|>0$. Since we are assuming that each $f_{k}$ is orientation-preserving, it follows that $f_{k}(\bar{x}) \in f_{k}\left(x_{i}\right) \curvearrowright f_{k}\left(x_{j}\right)$ for every $k \in \mathbb{N}$. Passing to the limit, we have therefore $\lim _{k \rightarrow \infty} f_{k}(\bar{x})=y_{i}$. Hence, once again by Remark 3.1, $0=\left|\varphi(\bar{x})-\psi\left(y_{i}\right)\right|=\left|\varphi(\bar{x})-\varphi\left(x_{i}\right)\right|>0$, leading to a contradiction.

In the sequel, for $i \neq 1, \ldots, n$, we assume that $x_{i}\left(\operatorname{resp} . y_{i}\right)$ is equal to $x_{(i \bmod n)}$ $\left(\right.$ resp. $\left.y_{(i \bmod n)}\right)$. 
The result below shows that each $y_{i}$ is a critical point of $\psi$ "of the same type" of the corresponding $x_{i}$.

Lemma 3.3. For every $i \in\{1, \ldots, n\}$, the following statements hold:

(i) If $x_{i}$ is a local minimum point of $\varphi$, then $y_{i}$ is a local minimum point of $\psi$.

(ii) If $x_{i}$ is a local maximum point of $\varphi$, then $y_{i}$ is a local maximum point of $\psi$.

Proof. Let us prove only $(i)$. Statement $(i i)$ can be verified analogously.

Let $x_{i}$ be a local minimum point of $\varphi$. Then $x_{i-1}$ and $x_{i+1}$ are local maximum points of $\varphi$, with $\varphi\left(x_{i}\right)<\varphi\left(x_{i-1}\right), \varphi\left(x_{i+1}\right)$. Since $\varphi\left(x_{j}\right)=\psi\left(y_{j}\right)$ for every index $j$, it follows that $\psi\left(y_{i}\right)<\psi\left(y_{i-1}\right), \psi\left(y_{i+1}\right)$. Moreover, since each $f_{k}$ is assumed to be orientation-preserving, if $x_{i} \in x_{i-1} \curvearrowright x_{i+1}$, then $f_{k}\left(x_{i}\right) \in f_{k}\left(x_{i-1}\right) \curvearrowright f_{k}\left(x_{i+1}\right)$ for every $k \in \mathbb{N}$. Hence, passing to the limit, it holds that $y_{i} \in y_{i-1} \curvearrowright y_{i+1}$, with $y_{i-1}, y_{i}, y_{i+1}$ distinct because of Lemma 3.2.

Contrary to our assertion, let us now suppose that $y_{i}$ is not a local minimum point of $\psi$. Then, we can find a point $\bar{y} \in y_{i-1} \curvearrowright y_{i+1}$ such that $\psi(\bar{y})<\psi\left(y_{i}\right)<$ $\psi\left(y_{i-1}\right), \psi\left(y_{i+1}\right)$. Consequently, there exists $\bar{k} \in \mathbb{N}$ such that, for every $k \geq \bar{k}, \bar{y} \in$ $f_{k}\left(x_{i-1}\right) \curvearrowright f_{k}\left(x_{i+1}\right)$, implying that, by the orientation-preservation assumption, $f_{k}^{-1}(\bar{y}) \in x_{i-1} \curvearrowright x_{i+1}$ for every $k \geq \bar{k}$. Possibly by considering a subsequence of $\left(f_{k}^{-1}\right)$, we can assume that $\left(f_{k}^{-1}(\bar{y})\right)$ converges. Let $\bar{x}=\lim _{k \rightarrow \infty} f_{k}^{-1}(\bar{y})$. Then $\bar{x} \in x_{i-1} \curvearrowright x_{i+1}$. Therefore $\varphi(\bar{x}) \geq \varphi\left(x_{i}\right)=\psi\left(y_{i}\right)>\psi(\bar{y})$, leading to $0=$ $\lim _{k \rightarrow \infty}\left|\varphi\left(f_{k}^{-1}(\bar{y})\right)-\psi(\bar{y})\right|=|\varphi(\bar{x})-\psi(\bar{y})|>0$, thus getting a contradiction (cf. Remarks 1.5 and 3.1).

We observe that $\psi$ does not admit any other critical point besides $y_{1}, \ldots, y_{n}$. Indeed, let us suppose that $\psi$ has $m$ critical points, $m>n$, and that (possibly by extracting a subsequence of $\left.\left(f_{k}\right)\right)\left(f_{k}^{-1}\right)$ converges at all such points. Then we can apply Lemmas 3.2 and 3.3 by interchanging the roles of $X$ and $Y$. In this way, we obtain that the number of critical points of $\varphi$ is not smaller than $m$, leading to a contradiction.

We are now ready to give the main result of this paper.

Theorem 3.4. Let $(X, \varphi),(Y, \psi)$ be two size pairs, with $X, Y$ closed curves, and $\varphi: X \rightarrow \mathbb{R}, \psi: Y \rightarrow \mathbb{R}$ Morse measuring functions such that $\delta((X, \varphi),(Y, \psi))=0$. Then there exists an optimal $C^{2}$-diffeomorphism $f: X \rightarrow Y$.

Proof. Let us define $f: X \rightarrow Y$ as follows:

- $f\left(x_{i}\right)=y_{i}$;

- for every $x \in x_{i} \curvearrowright x_{i+1}, f(x)=y$ with $y \in y_{i} \curvearrowright y_{i+1}$ and $\psi(y)=\varphi(x)$.

Let us prove that $f$ is well defined and bijective on $X$. Since the restriction of $f$ to $\left\{x_{1}, \ldots, x_{n}\right\}$ is injective because of Lemma 3.2 and the sets of critical points of $\varphi$ and $\psi$ have the same cardinality, it is sufficient to show that, for every index $i$, if $\varphi$ is strictly increasing (resp. decreasing) on the arc $x_{i} \curvearrowright x_{i+1}$, then $\psi$ is strictly increasing (resp. decreasing) on the arc $y_{i} \curvearrowright y_{i+1}$.

Let $\varphi$ be strictly increasing on the arc $x_{i} \curvearrowright x_{i+1}$ (the other case can be shown similarly). Under our assumptions, we have $\varphi\left(x_{i}\right)<\varphi\left(x_{i+1}\right)$, with $x_{i}$ a local minimum point and $x_{i+1}$ a local maximum point of $\varphi$. Therefore, by Remark 3.1, it follows that $\psi\left(y_{i}\right)<\psi\left(y_{i+1}\right)$, and by Lemma 3.3, $y_{i}$ is a local minimum point 
and $y_{i+1}$ is a local maximum point of $\psi$. By contradiction, let us suppose that $\psi$ is not strictly increasing on the arc $y_{i} \curvearrowright y_{i+1}$. Since $\psi$ is Morse on the curve $Y$, there exists at least one index $\bar{\jmath} \in\{1, \ldots, n\}, \bar{\jmath} \neq i, i+1$, such that the critical point $y_{\bar{\jmath}}$ of $\psi$ belongs to $y_{i} \curvearrowright y_{i+1}$. Then, the critical point $x_{\bar{\jmath}}$ of $\varphi$ is such that $\lim _{k \rightarrow \infty} f_{k}\left(x_{\bar{\jmath}}\right)=y_{\bar{\jmath}}$, but $x_{\bar{\jmath}} \notin x_{i} \curvearrowright x_{i+1}$. This means that there exists $\bar{k} \in \mathbb{N}$ such that, for every $k \geq \bar{k}, f_{k}\left(x_{\bar{\jmath}}\right) \in f_{k}\left(x_{i}\right) \curvearrowright f_{k}\left(x_{i+1}\right)$, but $x_{\bar{\jmath}} \notin x_{i} \curvearrowright x_{i+1}$. Hence, we get an absurd because each $f_{k}$ is a homeomorphism.

Let us observe that, by construction, $f$ is a homeomorphism, and it attains the natural pseudo-distance, that is $\Theta(f)=0$.

To conclude the proof, it remains to show that $f$ is locally a $C^{2}$-diffeomorphism.

For every $x \in X \backslash\left\{x_{1}, \ldots, x_{n}\right\}$, there exists a sufficiently small neighborhood $U$ of $x$ in $X$ such that $\varphi_{\left.\right|_{U}}$ and $\psi_{\left.\right|_{f(U)}}$ are $C^{2}$-diffeomorphism. Then $\varphi=\psi \circ f$ throughout $U$ implies $f=\psi^{-1} \circ \varphi$ throughout $U$. As a consequence, $f$ is a $C^{2}$-diffeomorphism on $U$.

If $x=x_{i}, i \in\{1, \ldots, n\}$, then, by the well-known Morse Lemma [26], there is a local coordinate system $s$ in an open arc $U$ of $X$ containing $x$, and a $C^{2}$ diffeomorphism $h_{\varphi}: U \rightarrow \mathbb{R}$ such that $h_{\varphi}(x)=0$ and $\varphi \circ h_{\varphi}^{-1}(s)=\varphi(x) \pm s^{2}$ throughout $h_{\varphi}(U)$. Moreover, $f(x)=y_{i}$. Therefore, in an open arc $V$ of $Y$ containing $f(x)$ there is a $C^{2}$-diffeomorphism $h_{\psi}: V \rightarrow \mathbb{R}$ such that $h_{\psi}(f(x))=0$ and $\psi \circ h_{\psi}^{-1}(s)=\psi(f(x)) \pm s^{2}$ throughout $h_{\psi}(V)$. Let $h_{\varphi}(U) \cap h_{\psi}(V)$ be an open interval $(a, b)$, with $0 \in(a, b)$. By the assumption $\delta((X, \varphi),(Y, \psi))=0$, necessarily $\varphi(x)=\psi(f(x))$, and by Lemma 3.3, either $\varphi \circ h_{\varphi}^{-1}(s)=\psi \circ h_{\psi}^{-1}(s)=\varphi(x)+s^{2}$ throughout $(a, b)$ (in the case $x$ and $f(x)$ are minima of $\varphi$ and $\psi$, respectively), or $\varphi \circ h_{\varphi}^{-1}(s)=\psi \circ h_{\psi}^{-1}(s)=\varphi(x)-s^{2}$ throughout $(a, b)$ (in the case $x$ and $f(x)$ are maxima of $\varphi$ and $\psi$, respectively). In both cases, $f=\psi^{-1} \circ \varphi=h_{\psi}^{-1} \circ h_{\varphi}$ throughout $h_{\varphi}^{-1}((a, 0))$ and $h_{\varphi}^{-1}((0, b))$ since $\psi$ is invertible in $h_{\psi}^{-1}((a, 0))$ and $h_{\psi}^{-1}((0, b))$, respectively. By continuity, $f=h_{\psi}^{-1} \circ h_{\varphi}$ throughout $h_{\varphi}^{-1}((a, b))$, i.e. $f$ is a $C^{2}$ diffeomorphism on $h_{\varphi}^{-1}((a, b))$.

As an immediate consequence of the previous theorem, we have the following Corollary 3.5, establishing a necessary and sufficient condition so that the natural pseudo-distance between two size pairs vanishes.

Corollary 3.5. Let $(X, \varphi),(Y, \psi)$ be two size pairs, with $X, Y$ closed curves, and $\varphi: X \rightarrow \mathbb{R}, \psi: Y \rightarrow \mathbb{R}$ Morse measuring functions. Then $\delta((X, \varphi),(Y, \psi))=0$ if and only if there exists a $C^{2}$-diffeomorphism $f: X \rightarrow Y$ such that $\varphi=\psi \circ f$.

Proof. The direct statement immediately follows from Theorem 3.4. Conversely, if such a diffeomorphism $f$ exists, then, by Definition $1.1, \Theta(f)=0$. Since $0 \leq$ $\delta((X, \varphi),(Y, \psi)) \leq \Theta(f)$, the claim is proved.

Figure 4 shows an example of how Corollary 3.5 can be used to decide if different closed curves have "the same shape" when compared with respect to a particular property (i.e., when a particular measuring function is considered).

For every $i=1, \ldots, 4$, the plane curve $\mathcal{C}_{i}$ is endowed with the Morse measuring function $\varphi_{i}$, taking each point of $\mathcal{C}_{i}$ to its Euclidean distance from the fixed point $p \in \mathbb{R}^{2}$. The critical points of $\varphi_{i}, i=1, \ldots, 4$, are displayed in figure. The size pairs are chosen in such a way that the natural pseudo-distance $\delta\left(\left(\mathcal{C}_{i}, \varphi_{i}\right),\left(\mathcal{C}_{j}, \varphi_{j}\right)\right)$ is equal to 0 for every $i, j=1, \ldots, 4$. Indeed, as in the proof of Theorem 3.4 , we 

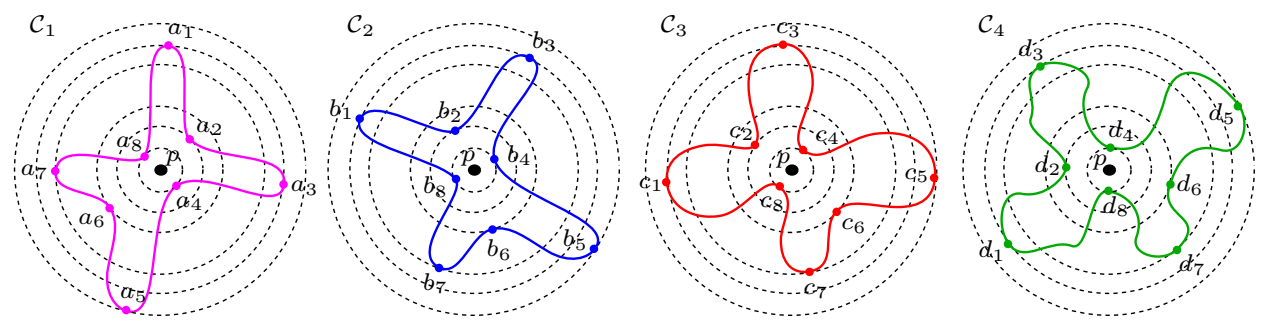

FigURE 4. Four size pairs $\left(\mathcal{C}_{i}, \varphi_{i}\right)$, with $\varphi_{i}: \mathcal{C}_{i} \rightarrow \mathbb{R}, \varphi_{i}(x)=\|x-p\|$ for every $x \in \mathcal{C}_{i}$. For each two of these pairs, there exists an optimal diffeomorphism, defined as in the proof of Theorem 3.4.

can easily define diffeomorphisms $f_{i j}: \mathcal{C}_{i} \rightarrow \mathcal{C}_{j}$ by matching each critical point of $\varphi_{i}$ with the corresponding critical point of $\varphi_{j}$, and then extending linearly such a correspondence. Obviously, $f_{i j}$ preserves the measuring functions' values, hence, by Corollary 3.5, $\delta\left(\left(\mathcal{C}_{i}, \varphi_{i}\right),\left(\mathcal{C}_{j}, \varphi_{j}\right)\right)=0$.

In this way, we could say that the four closed curves share the same shape property that is described by the chosen measuring functions and is characterized by the presence of 4 humps.

We observe that the shape property described in this example is invariant under rotations and reflections of the curves with respect to the point $p$, and under nonrigid deformations preserving the local minimum and maximum values, as well as the relative order of critical points of the associated measuring functions. This last invariance property could be particularly useful in Computer Vision applications, since it usually happens that two shapes can be perceived as similar even if they are non-rigidly related.

3.1. A topological interpretation of Theorem 3.4. From a topological point of view, our main result can be used to characterize the Morse functions on the circle $S^{1}$ up to the composition with $C^{2}$-diffeomorphisms of $S^{1}$ that do not change functions' values. Indeed, if we replace $X$ and $Y$ by $S^{1}$ in Theorem 3.4, we can consider the set of Morse functions on $S^{1}, \mathcal{M}\left(S^{1}, \mathbb{R}\right)$, endowed with the uniform convergence topology. Then, we can quotient such a space defining the following equivalence relation $\sim$ : for every $\varphi, \psi \in \mathcal{M}\left(S^{1}, \mathbb{R}\right), \varphi \sim \psi$ if there exists a diffeomorphism $f: S^{1} \rightarrow S^{1}$ such that $\varphi=\psi \circ f$. In this way, we can rephrase Theorem 3.4 as follows.

Theorem 3.4 (restated). The equivalence classes defined by the relation $\sim$ are closed in $\mathcal{M}\left(S^{1}, \mathbb{R}\right)$.

As an immediate consequence, we have that the natural pseudo-distance induces a distance $\widetilde{\delta}$ on the space $\mathcal{M}\left(S^{1}, \mathbb{R}\right) / \sim$, by setting $\widetilde{\delta}([\varphi],[\psi])=\delta\left(\left(S^{1}, \varphi\right),\left(S^{1}, \psi\right)\right)$ for every $[\varphi],[\psi] \in \mathcal{M}\left(S^{1}, \mathbb{R}\right) / \sim$.

\section{Conclusions and future Works}

In this paper we have proved that there always exists an optimal diffeomorphism between two size pairs $(X, \varphi),(Y, \psi)$ having vanishing natural pseudo-distance, under the assumptions that $X, Y$ are closed curves, and $\varphi, \psi$ are Morse measuring functions. We point out that this result is the first available one concerning the existence of optimal homeomorphisms between size pairs. Indeed, until now the 
research has been developed mainly focusing on the relations between the natural pseudo-distance and the measuring functions' critical values, as well as on the estimation of the natural pseudo-distance via lower bounds provided by size functions. Our result opens the way to further investigations, in order to obtain a generalization to the case of $n$-dimensional manifolds, $n>1$. To this end, as shown in Example 2, it is clear that we should consider measuring functions either satisfying more restrictive conditions (e.g. simple Morse functions), or taking values in $\mathbb{R}^{m}$, with $1<m \leq n$ (in general, we are not interested in the case $m>n$ since, at least in the perspective of shape comparison, this would increase the dimensionality of the problem). In the latter case, an interesting research line appears to be, for example, to consider measuring functions having finite preimage for each point in the range, or characterized by a behavior analogous to that of Morse functions in the 1-dimensional case.

Acknowledgments. The authors wish to thank P. Frosini for suggesting the problem and for his indispensable support and friendship. Moreover, they gratefully acknowledges F. Cagliari for several helpful comments and stimulating conversations. However, the authors are solely responsible for any possible errors.

\section{REFERENCES}

1. S. Biasotti, L. De Floriani, B. Falcidieno, P. Frosini, D. Giorgi, C. Landi, L. Papaleo, and M. Spagnuolo, Describing shapes by geometrical-topological properties of real functions, ACM Comput. Surv. 40 (2008), no. 4, 1-87.

2. S. Biasotti, D. Giorgi, M. Spagnuolo, and B. Falcidieno, Size functions for comparing 3D models, Pattern Recogn. 41 (2008), no. 9, 2855-2873.

3. G. Carlsson, Topology and data, Bull. Amer. Math. Soc. 46 (2009), no. 2, 255-308.

4. A. Cerri, B. Di Fabio, M. Ferri, P. Frosini, and C. Landi, Betti numbers in multidimensional persistent homology are stable functions, Technical report, Univ. of Bologna, 2010, http://amsacta.cib.unibo.it/2923/.

5. A. Cerri, M. Ferri, and D. Giorgi, Retrieval of trademark images by means of size functions, Graph. Models 68 (2006), no. 5, 451-471.

6. M. d'Amico, P. Frosini, and C. Landi, Natural pseudo-distance and optimal matching between reduced size functions, Acta Applicandae Mathematicae 109 (2010), no. 2, 527-554.

7. B. Di Fabio and C. Landi, Reeb graphs of curves are stable under function perturbations, Technical report, Univ. of Bologna, 2011, http://amsacta.cib.unibo.it/2998/.

8. F. Dibos, P. Frosini, and D. Pasquignon, The use of size functions for comparison of shapes through differential invariants, J. Math. Imaging Vis. 21 (2004), no. 2.

9. P. Donatini and P. Frosini, Lower bounds for natural pseudodistances via size functions, Archives of Inequalities and Applications 1 (2004), no. 2, 1-12.

10. N_ Natural pseudodistances between closed manifolds, Forum Mathematicum 16 (2004), no. 5, 695-715.

11. _ Natural pseudodistances between closed surfaces, Journal of the European Mathematical Society 9 (2007), no. 2, 231-253.

12. _ Natural pseudo-distances between closed curves, Forum Mathematicum 21 (2009), no. 6, 981-999.

13. P. Donatini, P. Frosini, and C. Landi, Deformation energy for size functions, EMMCVPR '99: Proceedings of the Second International Workshop on Energy Minimization Methods in Computer Vision and Pattern Recognition (London, UK), Springer-Verlag, 1999, pp. 44-53.

14. P. Frosini, A distance for similarity classes of submanifolds of a Euclidean space, Bulletin of the Australian Mathematical Society 42 (1990), no. 3, 407-416.

15. __ Measuring shapes by size functions, Society of Photo-Optical Instrumentation Engineers (SPIE) Conference Series (D. P. Casasent, ed.), Presented at the Society of PhotoOptical Instrumentation Engineers (SPIE) Conference, vol. 1607, February 1992, pp. 122-133. 
16. P. Frosini and C. Landi, Size theory as a topological tool for computer vision, Pattern Recognition and Image Analysis 9 (1999), 596-603.

17. Size functions and formal series, Appl. Algebra Engrg. Comm. Comput. 12 (2001), no. 4, 327-349.

18. P. Frosini and M. Mulazzani, Size homotopy groups for computation of natural size distances, Bulletin of the Belgian Mathematical Society 6 (1999), no. 3, 455-464.

19. R. Ghrist, Barcodes: the persistent topology of data, Bull. Amer. Math. Soc. (N.S.) 45 (2008), no. $1,61-75$.

20. D. Groisser, Certain optimal correspondences between plane curves, I: manifolds of shapes and bimorphisms, Trans. Amer. Math. Soc. 361 (2009), no. 6, 2959-3000.

21. __ Certain optimal correspondences between plane curves, II: existence, local uniqueness, regularity, and other properties, Trans. Amer. Math. Soc. 361 (2009), no. 6, 3001-3030.

22. T. Kaczynski, K. Mischaikow, and M. Mrozek, Computational homology, 1 ed., Applied Mathematical Sciences, no. 157, Springer-Verlag, 2004

23. C. Landi and P. Frosini, New pseudodistances for the size function space, Society of PhotoOptical Instrumentation Engineers (SPIE) Conference Series (\& L. J. Latecki R. A. Melter, A. Y. Wu, ed.), Presented at the Society of Photo-Optical Instrumentation Engineers (SPIE) Conference, Vision Geometry VI, vol. 3168, 1997, pp. 52-60.

24. P. W. Michor and D. Mumford, Riemannian geometries on spaces of plane curves, J. Eur. Math. Soc. (JEMS) 8 (2006), 1-48.

25. __ An overview of the riemannian metrics on spaces of curves using the hamiltonian approach, Appl. Comput. Harmon. Anal. 23 (2007), no. 1, 74-113.

26. J. Milnor, Morse theory, Based on lecture notes by M. Spivak and R. Wells. Annals of Mathematics Studies, no. 51, Princeton University Press, Princeton, N.J., 1963.

27. I. Stanganelli, A. Brucale, L. Calori, R. Gori, A. Lovato, S. Magi, B. Kopf, R. Bacchilega, V. Rapisarda, A. Testori, P.A. Ascierto, E. Simeone, and M. Ferri, Computer-aided diagnosis of melanocytic lesions, Anticancer Research 25 (2005), 4577-4582.

28. C. Uras and A. Verri, Computing size functions from edge maps, Int. J. Comput. Vision 23 (1997), no. 2 .

29. A. Zomorodian, Topology for computing, Cambridge Monographs on Applied and Computational Mathematics, vol. 16, Cambridge University Press, Cambridge, 2005.

ARCES, Università di Bologna, via Toffano 2/2, I-40135 Bologna, Italia

Dipartimento di Matematica, Università di Bologna, P.zza di Porta S. Donato 5, I-40126 Bologna, ItAlia

Current address: Pattern Recognition and Image Processing Group, Faculty of Informatics, Vienna University of Technology, Austria

E-mail address: \{cerri,difabio\}@dm.unibo.it, acerri@prip.tuwien.ac.at 